\title{
Nukleäre Rezeptoren beim hepatischen und intestinalen Medikamententransport
}

Die Bioverfügbarkeit von Pharmaka hängt vom Ausmaß der Resorption im Darm, der Biotransformation und dem Eliminationsweg ab. Die Resorption im Darm erfolgt über Transportproteine der Lamina epithelialis mucosae. Die Biotransformation beginnt bereits in intestinalen Epithelzellen und die aufgenommenen Xenobiotika werden über Effluxpumpen der basalen oder apikalen Membran in die Blutbahn bzw. zurück in das Darmlumen abgegeben [1]. Das Pfortaderblut erreicht die basolaterale (sinusoidale) Hepatozytenmembran, die ihrerseits mit multiplen Transportsystemen ausgestattet ist. Xenobiotika werden in unterschiedlichem Ausmaß von Hepatozyten aufgenommen und in Phase-1/II-Reaktionen biotransformiert. Die entstandenen polaren Medikamentenmetaboliten können über Effluxpumpen der basolateralen oder kanalikulären (apikalen) Hepatozytenmembran in das venöse Blut bzw. in die Galle ausgeschieden werden.

Nukleäre Rezeptoren sind Liganden-aktivierte Transkriptionsfaktoren, welche durch ein breites Spektrum von Xenobiotika und endogenen lipophilen Substanzen aktiviert werden. Das humane Genom enthält 48 Mitglieder dieser Gen-Superfamilie [2], welche die Subfamilien der Steroidhormonrezeptoren und der „orphanen“ nukleären Rezeptoren umfasst. Letztere werden in „adoptierte“ orphane nukleäre Rezeptoren umbenannt, sobald ein endogener Ligand bekannt ist. Daher werden endokrine, adoptierte orphane und orphane nukleäre Rezeptoren unterschieden, je nach physiologischem Liganden und potentieller Funktion [3]. Nukleäre Steroidhormonrezeptoren, wie der Glukokortikoid-Rezeptor (GR), MineralokortikoidRezeptor (MR), Östrogen-Rezeptor (ER), Androgen-Rezeptor (AR) und Progesteron-Rezeptor (PR), binden DNA als Homodimere und ihre Liganden sind endogene Steroidhormone. Orphane nukleäre Rezeptoren binden DNA als Heterodimere mit dem Retinoid-X-Rezeptor (RXR). Zu den wichtigsten Liganden zählen Fettsäuren (für PPAR $\alpha$, den „peroxisome proliferator activated receptor“), Oxysterole (für LXR, den „liver $X$ receptor“), Gallensäuren (für FXR, den „farnesoid $X$ receptor“, PXR, den , pregnane X receptor“, und VDR, den Vitamin-D-Rezeptor) und Xenobiotika (für PXR und CAR, den „constitutive androstane receptor“). Nukleäre Rezeptoren sind entscheidende Regulatoren der entgiftenden Enzym- und Transportsysteme in der Leber und im Darm, die durch Xenobiotika und endogene lipophile Substanzen aktiviert werden.

\section{Intestinaler Medikamenten- transport}

Die Menge eines Pharmakons, die nach oraler Zufuhr vom Darmlumen in das Pfortaderblut übertritt, wird maßgeblich durch den präsystemischen Stoffwechsel im Enterozyten bestimmt. Die Aufnahme eines Medikaments erfolgt über Importsysteme der luminalen Enterozytenmembran, z. B. über den „apical sodium-dependent bile salt transporter" ASBT (Gensymbol: SLC10A2) oder über OATPs („organic anion transporting polypeptides“, z.B. SLCO1A2 oder SLCO2B1), um nur zwei der besser charakterisierten Transporterklassen zu nennen [4]. Geschwindigkeitsbestimmend ist jedoch weniger die Aufnahme als der Efflux, z. B. über das MDR1P-Glykoprotein (,multidrug resistance gene product“), welches Xenobiotika aus dem Zellinneren zurück in das Darmlumen transportiert. Dieser intestinale Efflux ist eine kritische Determinante der Bioverfügbarkeit eines Medikaments. Digoxin, Ciclosporin und Paclitaxel sind prototypische Substrate der Effluxpumpe MDR1 im Darm. In Probandenstudien wurde beobachtet, dass die Bioverfügbarkeit von per os zugeführtem Digoxin nach 10-tägiger Einnahme von Rifampicin deutlich abnahm [5]. In Duodenalbiopsien der Rifampicin-behandelten Probanden fand sich eine 3,5-fache Zunahme der MDR1-Proteinmenge, was mit früheren Untersuchungen an Kolonkarzinom-Zellinien übereinstimmte, die eine vermehrte MDR1-Expression nach Rifampicin-Behandlung aufwiesen [6]. Die Abnahme der Bioverfügbarkeit von Digoxin erklärt sich durch die Induktion des MDR1-vermittelten Auswärtstransports von Digoxin zurück in das Darmlumen. Der Mechanismus der Rifampicininduzierten Steigerung der MDR1-Expres- sion ist eine direkte transkriptionelle Aktivierung des MDR1-Gens durch den nukleären Rezeptor PXR [7]. PXR ist an ein DR-4 („direct repeat“) Element des MDR1-Gens etwa 8000 Basen vor der Gentranskriptions-Initiationsstelle gebunden und aktiviert, nach Bindung des Liganden Rifampicin, die Gentranskription. Zu den Liganden des humanen PXR gehören z. B. Rifampicin, Antiglucocorticoide, Dexamethason, Johanniskrautextrakt und Paclitaxel [8].

Die zentrale Bedeutung von PXR als Aktivator des Xenobiotika-Abbaus wurde anhand der PXR-vermittelten Induktion des Cytochroms P450 3A4 (CYP3A4) erkannt. Die CYP3A4-Gentranskription wird durch einen Liganden des PXR induziert, welcher als Heterodimer mit PXR an das „xenobiotic response element" im Promotor des CYP3A4 Gens bindet [9]. PXR wird ähnlich wie CYP3A4 hauptsächlich in der Leber und im Darm exprimiert. Viele Substanzen, die durch CYP3A4 metabolisiert werden, sind auch Substrate von MDR1. Eine koordinierte Induktion beider Gene erhöht daher die Effizienz des Abbaus und der Elimination der betreffenden Substanzen. Bestimmte CYP3A4/MDR1-Substrate sind darüber hinaus noch Liganden des PXR, wodurch sie ihren eigenen Abbau induzieren können. Ein Beispiel hierfür ist das Chemotherapeutikum Paclitaxel [10]. Paclitaxel ist ein Substrat von CYP3A4 und MDR1 und induziert die Gentranskription beider Proteine über eine Aktivierung von PXR.

\section{Hepatischer Medikamenten- transport}

Zu den wichtigsten Xenobiotika-Effluxpumpen der Leber zählen die MRPs („,multidrug resistance associated proteins“). Eine Sonderstellung nimmt hierbei das MRP2 ein ( $A B C C 2)$, da es als einziges an der kanalikulären Hepatozytenmembran lokalisiert ist, während MRP3, 4, 5 und 6 basolateral exprimiert sind. MRP2 ist ein aktives Effluxsystem für anionische Konjugate, wie z.B. Bilirubinglukuronide, sulfatierte Gallensäuren und Glutathionkonjugate, sowie für Xenobiotika wie Cisplatin, HIV-Proteaseinhibitoren, Anthrazykli- 
ne, Vinca-Alkaloide und Methotrexat [11]. MRP2 wird auch im Darm exprimiert - an der apikalen Membran von Enterozyten [12]. Das für MRP2 kodierende Gen, $A B C C 2$, wird durch PXR, CAR und FXR induziert [11]. Das an der basolateralen Membran exprimierte MRP3 ist für den Efflux von Methotrexat, Acetaminophen-Glukuroniden, Bilirubin, Östradiol-17ß-Glukuronid, Ritonavir, Saquinavir, konjugierter Cholsäure und diversen weiteren Substraten aus dem Hepatozyten ins sinusoidale Blut verantwortlich [11]. Auch MRP3 scheint durch PXR, CAR und FXR induzierbar zu sein [13].

Zu den wichtigsten Aufnahmetransportern für Medikamente an der basolateralen Hepatozytenmembran zählen die OATPs, insbesondere OATP1B1 und OATP1B3. OATP1B1 weist eine sehr breite Substratspezifität auf, zum Beispiel für Statine, Methotrexat, Olmesartan und viele andere [11]. OATP1B3 ist ein Aufnahmetransporter für Digoxin [4], aber auch für bestimmte Peptide wie Cholecystokinin [14]. Im Gegensatz zum Digoxintransporter Oatp1a4 der Ratte wird der Promotor des humanen OATP1B3 Gens nicht durch PXR, sondern durch FXR aktiviert [15]. Die Liganden von FXR sind in erster Linie Gallensäuren, so auch die synthetische Gallensäure Obeticholsäure, welche als Therapie der primär biliären Cholangitis in Kombination mit Ursodeoxycholsäure zugelassen ist. FXR induziert zudem die „bile salt export pump“ an der kanalikulären Hepatozytenmembran (BSEP, ABCB11 Gen) [16]. Durch die koordinierte Induktion von Gallensäurenaufnahme- und -effluxtransportern führt der FXR-Ligand Obeticholsäure zu einer Steigerung der biliären Ausscheidung von Gallensäuren [17]. Auch der an der basolateralen Hepatozytenmembran exprimierte heterodimere Effluxtransporter „organic solute transporters alpha and beta“ (OST $\alpha /$ OST $\beta$ ), welcher primär Gallensäuren aus dem Hepatozyten ins sinusoidale Blut transportiert, wird durch FXR induziert [18].

Zusammengefasst, beeinflusst der hepatische Stoffwechsel massgeblich die Bioverfügbarkeit eines Medikaments. Die betei- ligten Transport- und Enzymsysteme des Hepatozyten werden durch nukleäre Rezeptoren wie PXR, CAR oder FXR reguliert. Die Verabreichung von Substanzen, die als Liganden für diese nukleären Rezeptoren dienen, kann den hepatischen Medikamententransport erheblich verändern und damit zu relevanten Medikamenteninteraktionen führen.

\section{Interessenkonflikt}

Aktien bei Novartis.

\section{Autor}

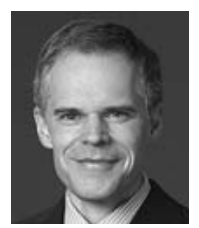

\section{Gerd A. Kullak-Ublick}

Prof. Dr. med., Klinikdirektor, Klinik für Klinische Pharmakologie und Toxikologie, UniversitätsSpital Zürich, Schweiz

\section{Korrespondenzadresse}

Prof. Dr. med. Gerd A. Kullak-Ublick

Klinikdirektor

Klinik für Klinische Pharmakologie

und Toxikologie

UniversitätsSpital Zürich

Rämistrasse 100

8091 Zürich

Schweiz

Tel.: + 41442552068

gerd.kullak@usz.ch

\section{Literatur}

[1] Kullak-Ublick GA, Jung D, Meier PJ. [Role of nuclear receptors in hepatic and intestinal drug transport]. Dtsch Med Wochenschr 2003; 128: 389-393

[2] Chawla A, Repa J], Evans RM et al. Nuclear receptors and lipid physiology: opening the $\mathrm{X}$ files. Science 2001; 294: 1866-1870

[3] Amacher DE. The regulation of human hepatic drug transporter expression by activation of xenobiotic-sensing nuclear receptors. Expert Opin Drug Metab Toxicol 2016; 12: 1463-1477

[4] Kullak-Ublick GA, Ismair MG, Stieger B et al. Organic anion-transporting polypeptide $B$ (OATP-B) and its functional comparison with three other OATPs of human liver. Gastroenterology 2001; 120: 525-533

[5] Greiner B, Eichelbaum M, Fritz P et al. The role of intestinal $\mathrm{P}$-glycoprotein in the inter- action of digoxin and rifampin. J Clin Invest 1999; 104: 147-153

[6] Schuetz EG, Beck WT, Schuetz JD. Modulators and substrates of P-glycoprotein and cytochrome P4503A coordinately up-regulate these proteins in human colon carcinoma cells. Mol Pharmacol 1996; 49: 311-318

[7] Geick A, Eichelbaum M, Burk O. Nuclear receptor response elements mediate induction of intestinal MDR1 by rifampin. J Biol Chem 2001; 276: 14581-14587

[8] Eloranta J], Meier PJ, Kullak-Ublick GA. Coordinate transcriptional regulation of transport and metabolism. Methods Enzymol 2005; 400: $511-530$

[9] Liddle C, Goodwin B. Regulation of hepatic drug metabolism: role of the nuclear receptors PXR and CAR. Semin Liver Dis 2002; 22: 115-122

[10] Synold TW, Dussault I, Forman BM. The orphan nuclear receptor SXR coordinately regulates drug metabolism and efflux. Nat Med 2001; 7: 584-590

[11] Staudinger JL, Woody S, Sun M et al. Nuclearreceptor-mediated regulation of drug- and bile-acid-transporter proteins in gut and liver. Drug Metab Rev 2013; 45: 48-59

[12] Tocchetti GN, Rigalli JP, Arana MR et al. Modulation of expression and activity of intestinal multidrug resistance-associated protein 2 by xenobiotics. Toxicol Appl Pharmacol 2016; 303: 45-57

[13] Guo GL, Lambert G, Negishi M et al. Complementary roles of farnesoid $X$ receptor, pregnane $X$ receptor, and constitutive androstane receptor in protection against bile acid toxicity. J Biol Chem 2003; 278: 45062-45071

[14] Ismair MG, Stieger B, Cattori $\vee$ et al. Hepatic uptake of cholecystokinin octapeptide by organic anion-transporting polypeptides OATP4 and OATP8 of rat and human liver. Gastroenterology 2001; 121: 1185-1190

[15] Jung D, Podvinec M, Meyer UA et al. Human organic anion transporting polypeptide 8 promoter is transactivated by the farnesoid $X$ receptor/bile acid receptor. Gastroenterology 2002; 122: 1954-1966

[16] Kullak-Ublick GA, Stieger B, Meier PJ. Enterohepatic bile salt transporters in normal physiology and liver disease. Gastroenterology 2004; 126: 322-342

[17] Kjaergaard K, Frisch K, Sorensen M et al. Obeticholic acid improves hepatic bile acid excretion in patients with primary biliary cholangitis. J Hepatol 2021; 74: 58-65

[18] Landrier JF, Eloranta JJ, Vavricka SR et al. The nuclear receptor for bile acids, FXR, transactivates human organic solute transporteralpha and -beta genes. Am J Physiol Gastrointest Liver Physiol 2006; 290: G476-G485 